\title{
The IGFR1 inhibitor NVP-AEW541 disrupts a pro-survival and pro-angiogenic IGF-STAT3-HIF1 pathway in human glioblastoma cells
}

\author{
Marzia B. Gariboldi, Raffaella Ravizza, Elena Monti*

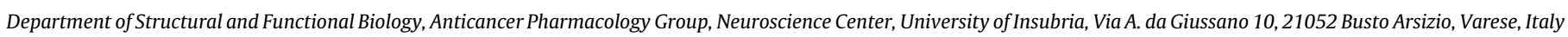

\section{A R T I C L E I N F O}

\section{Article history:}

Received 23 March 2010

Accepted 11 May 2010

\section{Keywords:}

Insulin-like growth factors

Hypoxia-inducible factor-1

STAT3

Glioblastoma

IGF-1 receptor inhibitor

\begin{abstract}
A B S T R A C T
Inappropriate activation of the IGF (insulin-like growth factor) system has been implicated in the growth and progression of a number of tumor types. Recent evidence indicates a possible role for the IGF system in modulating/mediating tumor cell response to hypoxia, a common occurrence in solid tumors, and particularly in malignant gliomas, causing tumor cells either to die, or to mount a pleiotropic adaptive response that is mainly orchestrated through activation of the hypoxia-inducible transcription factor HIF1. Experimental evidence suggests possible links between IGF- and HIF1-dependent signaling pathways, as well as a role for activated STAT3 in mediating their activities. Interestingly, igf2 is among the target genes transactivated by HIF1, thereby providing the missing link in a hypothetical autocrine self-amplifying circuit.

The present study investigates the presence of the IGF-HIF1-VEGF axis in the human glioma cell line U-87 MG, and characterizes its molecular effectors. Our results show that exogenous IGF-I causes IGF1R and STAT3 activation, and increases HIF1 $\alpha$ protein levels and HIF1 trascriptional activity, inducing VEGF release; a similar response, mediated by IGF-II release, is observed following HIF1 $\alpha$ stabilization. The existence of an autocrine loop is confirmed by its down-regulation following inactivation of IGF1R ( $u$ sing the IGF1R-specific tyrosine kinase inhibitor NVP-AEW541), STAT3 (transfecting the cells with an expression vector encoding a dominant negative form of STAT3), or HIF1 (using the small molecule inhibitor YC-1). The ability of NVP-AEW541 to block this circuit could be beneficial in suppressing the growth and angiogenic potential of hypoxic glial tumors.
\end{abstract}

(c) 2010 Elsevier Inc. All rights reserved.

\section{Introduction}

The existence of an autocrine loop involving the IGF (insulinlike growth factor) system and HIF1 (hypoxia-inducible factor 1) is emerging as a survival strategy common to different tumor types $[1,2]$. The IGF signaling pathway is activated by binding of one of two soluble polypeptide ligands (IGF-I and IGF-II) to the tyrosine kinase IGF1R, a tetrameric receptor consisting of two extracellular $\alpha$ chains and two transmembrane $\beta$ chains linked by a disulfide bond, bearing a high degree of homology with the insulin receptor (IR) [3]. Although IGF1R overexpression has been demonstrated in tumors, its activation is strictly dependent on ligand availability, which in turn can be regulated by the expression levels of the decoy receptor IGF2R (also known as the mannose-6-phosphate receptor) and of six IGF-binding proteins (IGFBPs). IGF1R activation has been implicated in several key features in malignancy, including loss of anchorage-dependent growth, evasion of

\footnotetext{
* Corresponding author. Tel.: +39 0331 339415; fax: +39 0331339459.

E-mail addresses: marzia.gariboldi@uninsubria.it (M.B. Gariboldi), raffaella.ravizza@gmail.com (R. Ravizza), elena.monti@uninsubria.it (E. Monti).
}

apoptotic signals, proteolytic degradation of the extracellular matrix and tumor angiogenesis [4].

Interestingly, the IGF signaling pathway appears to be intimately connected with HIF1 activation, which indeed seems to mediate at least part of IGF-induced downstream effects. HIF1 is a heterodimeric transcription factor, the activity of which depends on the intracellular levels of its inducible $\alpha$ subunit. In the presence of oxygen, HIF $1 \alpha$ is hydroxylated on two critical proline residues $\left(\right.$ Pro $_{402}$ and Pro $\left._{564}\right)$ in the so-called oxygen-dependent degradation domain (ODDD). This modification targets the protein for ubiquitylation by an E3 ligase complex (including the Von Hippel-Lindau tumor suppressor protein) and subsequent proteasomal degradation [5]. Growth factor tyrosine kinase receptor activation has been reported to increase HIF1 $\alpha$ levels, as well as HIF1 activity, under both normoxic and hypoxic conditions, mostly through up-regulation of the PI3K/Akt and Ras/Raf/MAPK pathways [5,6]. HIF1 orchestrates a pleiotropic adaptive response to hypoxia by inducing the expression of more than 100 genes encoding glycolytic enzymes and glucose transporters (thereby facilitating the glycolytic switch in energy metabolism typically observed under hypoxic conditions), matrix metalloproteinases, and angiogenic as well as mitogenic and survival factors. Most notably, IGF-II is among the growth factors 
up-regulated by HIF1, thereby potentially participating in a tumor growth-promoting autocrine loop [7].

Members of the STAT (signal transducers and activators of transcription) family might also be involved in mediating signaling along an IGF-HIF1 axis. In unstimulated cells, STAT proteins are present in a latent, inactive form; upon engagement of cytokine and growth factor receptors by their respective ligands, STAT proteins are recruited to activated receptor complexes and phosphorylated on tyrosine residues, whereby they dimerize and translocate to the nucleus, to modulate target gene expression [8]. Constitutive activation of STAT proteins, and particularly of STAT3, has been demonstrated in a number of cancer cell lines and tumors, including gliomas $[9,10]$, presumably due to dysregulation of growth factor receptor expression and/or activation. However, the role played by STAT3 in IGF signaling has only been investigated superficially $[11,12]$.

Conditions required to activate a putative regulatory IGF-HIF1 loop at different points are common in solid tumors. Regions of intermittent or chronic hypoxia frequently develop within the tumor mass, due to the fact that proliferating cells rapidly outgrow existing vessels and to the aberrant function of newly formed tumor vessel; hypoxia-induced IGF-II release might then sustain the growth of IGF1R expressing tumors. Similarly, when the circuit is activated by increased availability of IGFs, the downstream effects of IGF1R might be greatly amplified through HIF1 activation.

A number of strategies have been devised to disrupt the IGF signaling axis, ranging from forced expression of M6P/IGF2R or IGFBPs to the development of small interfering RNAs, antisense oligonucleotides, monoclonal antibodies and catalytic inhibitors targeting IGF1R [13-15]. Similarly, HIF1 has also been considered as a therapeutic target [16,17]: several small molecule inhibitors have been described [17]; an antisense oligonucleotide targeting HIF1 $\alpha$ mRNA, as well as a small molecule inhibitor (PX-478) are currently undergoing phase I clinical trials [18,19]. Merging these two approaches might lead to mutual potentiation of their effects in tumors expressing an overactive IGF-HIF1 circuit.

Based on these premises, the present study investigates the role played by the IGF-HIF1-VEGF axis in a human glioma cell line, and its possible regulation through an autocrine circuit. Gliomas are the most common neoplasms in the adult human brain and they are frequently characterized by coexistence of intense neoangiogenesis and hypoxic and necrotic regions; in addition, the IGF system has been implicated in tumorigenesis in the CNS, and particularly in the pathogenesis of glial tumors $[13,20]$. Our results indicate that: (a) an autocrine loop, involving IGF1R, STAT3, HIF1 and IGF-II, exists in U-87 MG cells, regulating tumor cell survival and VEGF production; and (b) the circuit can be effectively disrupted by inhibiting IGF1R activation with the selective pyrrolo[2,3-d]pyrimidine derivative NVP-AEW541 [21], which reduces both cell viability and VEGF release. These observations suggest that pharmacological targeting of the IGF1R may provide a significant therapeutic benefit, especially in hypoxic tumors featuring autocrine regulation of the IGF-HIF1 axis.

\section{Materials and methods}

\subsection{Reagents}

Standard chemicals and cell culture reagents were purchased from Sigma-Aldrich srl. (Milan, Italy), unless otherwise indicated; NVP-AEW541 was generously provided by Novartis (Basel, Switzerland).

\subsection{Cell culture, cytotoxicity studies and HIF1 $\alpha$ stabilization}

The human glioblastoma cell line U-87 MG (ATCC ${ }^{\circledR}$ HTB$14^{\mathrm{TM}}$ ) was obtained from the American Type Culture Collection
(Manassas, VA, USA), and authenticated by morphological inspection, growth curve analysis and short tandem repeat profiling, using the Promega PowerPlex ${ }^{\circledR} 1.2$ system (Promega, Madison, WI, USA) and the Applied Biosystems Genotyper ${ }^{\circledR} 2.0$ software (Applied Biosystems, Foster City, CA, USA) for amplicon analysis. Cells were maintained in DMEM supplemented with $10 \%$ fetal bovine serum (Euroclone, Italy), 1\% glutamine, 1\% antibiotic mixture, $1 \%$ sodium pyruvate, $1 \%$ non-essential aminoacids, at $37{ }^{\circ} \mathrm{C}$ in a humidified $5 \% \mathrm{CO}_{2}$ atmosphere and were routinely checked for Mycoplasma infection, using the Mycoplasma Plus ${ }^{\mathrm{TM}}$ PCR Primer Set (Stratagene, Agilent Technologies, La Jolla, CA).

To assess cell survival following NVP-AEW541 exposure, $4 \times 10^{4} \mathrm{U}-87 \mathrm{MG}$ cells/well were seeded onto 24 -well plates and allowed to grow for $24 \mathrm{~h}$ before treatment with different NVPAEW541 concentrations $(0.25,0.5,1.0$ and $2.5 \mu \mathrm{M})$. After $24 \mathrm{~h}$ (and subsequently at 48 and $72 \mathrm{~h}$ for growth curves), cells from 3 replicate wells per treatment were detached by trypsinization and resuspended in PBS containing 0.2\% Trypan blue; viable cells were counted using a Bürker hemocytometer following $10 \mathrm{~min}$ incubation.

To achieve HIF1 $\alpha$ stabilization, cells were exposed to $50 \mu \mathrm{M}$ $\mathrm{CoCl}_{2}$ for $24 \mathrm{~h}$, a condition that mimics hypoxia by inhibiting prolyl hydroxylation of the ODDD and its subsequent interaction with the Von Hippel-Lindau protein [22].

\subsection{Vectors and transfections}

U-87 MG cells were transiently transfected with an expression plasmid (pEF-HA-STAT3F) containing a full-length cDNA encoding hemagglutinin peptide (HA)-tagged STAT3F, a dominant negative form of STAT3 in which $\mathrm{Tyr}_{705}$ is replaced by phenylalanine, subcloned into a pEF-BOS expression vector (originally provided by Prof. S. Nagata, University of Osaka Medical School, Japan [23]); control cells were transfected with the pEF-BOS plasmid. pEF-HA-STAT3F was a generous gift from Prof. T. Hirano (Department of Molecular Oncology, University of Osaka Medical School, Japan [24]). Log-phase growing cells $\left(5 \times 10^{6}\right)$ were harvested and transfected with $30 \mu \mathrm{g}$ of DNA, using an EasyJet electroporator system (Equibio, Ashford, UK), under conditions optimized according to the manufacturer's instructions $(200 \mathrm{~V}$, $1500 \mu \mathrm{F}$ ). All experiments were performed starting $48 \mathrm{~h}$ after transfection.

The pBabe-STAT3C expression vector, containing a cDNA encoding a constitutively activated form of STAT3, was a generous gift from J. Bromberg et al. [25] and was transfected into U-87 MG cells by lipofection, using Lipofectamine $2000^{\circledR}$ (Invitrogen, Carlsbad, CA, USA). Briefly, $1 \times 10^{6}$ cells were seeded onto $100 \mathrm{~mm}$ Petri dishes and allowed to grow for $24 \mathrm{~h}$ before lipofection with the pBabe-STAT3C plasmid; control cells were transfected with the "empty" pBabe plasmid. STAT3C expression in transfected cells persisted for 6-8 days; all the experiments were performed within 5 days after transfection.

U-87/HRP-EGFP cells were obtained from U-87 MG cells by transient transfection with a plasmid containing the EGFP (enhanced green fluorescent protein) cDNA under the control of an artificial hypoxia-responsive promoter (HRP), consisting of five copies of a 35-bp fragment from the HRE of the human VEGF gene and a human cytomegalovirus (CMV) minimal promoter (kindly provided by Dr. Y. Cao) [26]. Cells were seeded onto 6 -well plates $\left(3.5 \times 10^{5}\right.$ cells/well $)$ and allowed to attach for $24 \mathrm{~h}$ before lipofection using Lipofectamine $200{ }^{\circledR}$. EGFP expression in transfected cells (following HIF1 $\alpha$ stabilization with $\mathrm{CoCl}_{2}$ at $50 \mu \mathrm{M}$ for $24 \mathrm{~h}$ ) persisted for $6-8$ days; all the experiments were performed within 5 days after transfection. 


\subsection{Evaluation of STAT3 and HIF-1 trascriptional activities}

STAT3-dependent transcriptional activity was assessed in nuclear extracts obtained from U-87 MG (parental), U-87-STAT3C and U-87-pBabe cells, using the TransAM ${ }^{\mathrm{TM}}$ STAT3 Trascription Factor Assay Kit (Active Motif, Carlsbad, CA, USA) according to the manufacturers' instructions. HIF1-dependent transcriptional activity was determined in U-87/HRP-EGFP cells following exposure to $\mathrm{CoCl}_{2}(50 \mu \mathrm{M}$ for $24 \mathrm{~h})$ or IGF-I stimulation $(100 \mathrm{ng} / \mathrm{ml}$ for $15 \mathrm{~min}$ ), in the presence or absence of $1 \mu \mathrm{M}$ NVP-AEW541 for $24 \mathrm{~h}$. Cell samples were analyzed with a FACSCalibur ${ }^{\mathrm{TM}}$ flow cytometer (Becton Dickinson, Mountain View, CA, USA), equipped with a $15 \mathrm{~mW}, 488 \mathrm{~nm}$ and air-cooled argon ion laser. At least 10,000 events were analyzed for each sample and data were processed using CellQuest ${ }^{\mathrm{TM}}$ software (Becton Dickinson). EGFP fluorescence data were collected, using log amplification, in the FL1 channel (530/30) and fluorescence intensity was expressed as mean fluorescence channel (MFC).

\subsection{Immunoprecipitation and Western blot analysis}

Activation of IGF1R and of STAT3 was evaluated by immunoprecipitation of cell lysates obtained from U-87 MG, U-87 pEF-BOS and U-87 STAT3F cells, with or without exposure to IGF-I (100 ng/ $\mathrm{ml}$ for $15 \mathrm{~min}$ ) and/or NVP-AEW541 ( $1 \mu \mathrm{M}$ for $24 \mathrm{~h}$ ). Lysis buffer composition was as follows: $\mathrm{NaCl} 120 \mathrm{mM}$, NaF $25 \mathrm{mM}$, EDTA $5 \mathrm{mM}$, EGTA $6 \mathrm{mM}$, sodium pyrophosphate $25 \mathrm{mM}$, in Tris- $\mathrm{HCl}$ $50 \mathrm{mM}$ pH 7.5, containing PMSF $0.2 \mathrm{mM}, \mathrm{Na}_{3} \mathrm{VO}_{4} 1 \mathrm{mM}$, phenylarsine oxide $1 \mathrm{mM}, \mathrm{NP}-401 \%$ and protease inhibitor cocktail 10\%). Protein concentration was determined by the bicinchoninic acid method using the BCA ${ }^{\mathrm{TM}}$ Protein Assay Kit (Pierce, Rockford, IL, USA) and $1000 \mu \mathrm{g}$ of protein were incubated overnight with the primary antibody anti-IGF1R or anti-STAT3 (rabbit polyclonal antibodies, Santa Cruz Biotechnology, Inc., Santa Cruz, CA, USASCB) at $4{ }^{\circ} \mathrm{C}$ and immunoprecipitated following $2 \mathrm{~h}$ incubation with protein G-Sepharose ${ }^{\mathrm{TM}}$ (Sigma-Aldrich, Milan, Italy). Proteins were then dissolved in sample buffer and separated by SDS-PAGE, transferred onto Immobilon ${ }^{\mathrm{TM}}$-P PVDF membranes (Millipore, Bedford, MA, USA) and Western blot analysis was performed incubating the membranes with a mouse monoclonal anti-pTyr antibody (PY20, SCB) to evaluate the phosphorylation state of the receptor, or with a rabbit anti-pSTAT3 polyclonal antibody (Cell Signaling Technology, Danvers, MA, USA). Total IGF1R or STAT3 protein levels were obtained by incubating the same membranes with anti-IGF1R or anti-STAT3 rabbit polyclonal antibodies. Proteins were visualized using peroxidase-conjugated secondary antibodies and the SuperSignal ${ }^{\circledR}$ West Pico Chemiluminescent Substrate (Pierce).

The effects of IGF-I and/or NVP-AEW541 on HIF1 $\alpha$ expression in normoxia and following HIF $1 \alpha$ stabilization with $\mathrm{CoCl}_{2}(50 \mu \mathrm{M}$ for $24 \mathrm{~h}$ ) were determined by Western blot analysis of total cell lysates. Fifty microgram of proteins were separated and transferred onto Immobilon ${ }^{\mathrm{TM}}$-P membranes and the membranes were incubated with a mouse anti-HIF1 $\alpha$ monoclonal antibody (Becton Dickinson, San Jose, CA, USA). Equal loading of the samples was verified by re-probing the blots with a mouse monoclonal antiactin antibody (SCB).

\subsection{VEGF and IGF-II release}

VEGF levels were measured in supernatants from U-87 MG cell cultures following exposure to NVP-AEW541 (1.0 $\mu \mathrm{M}$ for $24 \mathrm{~h}$ ) and IGF-I $(100 \mathrm{ng} / \mathrm{ml}$ for $15 \mathrm{~min})$, using the Human ELISA VEGF Development Kit (PeproTech, Inc., Rocky Hill, NJ, USA), according to the manufacturer's instructions. IGF-II levels were measured in supernatants from U-87 MG cell cultures following 24-h exposure to $\mathrm{CoCl}_{2}(50 \mu \mathrm{M})$ and $\mathrm{YC}-1(10 \mu \mathrm{M})$ using a Human Total IGF-II DuoSet $^{\circledR}$ ELISA System (R\&D Systems, Inc., Minneapolis, MN). Growth factor release was quantitated colorimetrically using a Universal Microplate Reader EL800 (BioTek, Winooski, VT, USA) at $405 \mathrm{~nm}$ for VEGF and at $450 \mathrm{~nm}$ for IGF-II.

\subsection{Statistical analysis}

Statistical analysis of the data was performed by two-way ANOVA, with Bonferroni's post-test for multiple comparisons.

\section{Results}

3.1. IGF1R stimulation causes STAT3 activation, increases HIF1 $\alpha$ protein levels and HIF1 activity, and induces VEGF release

Fig. 1A shows the effects of exogenous IGF-I, with or without the IGFR1 inhibitor NVP-AEW541, on tyrosine phosphorylation of IGF1R and STAT3, and on HIF1 $\alpha$ protein levels, as assessed by immunoprecipitation and/or Western blot. Immunoreactive bands corresponding to all three proteins were detected in U-87 MG cells

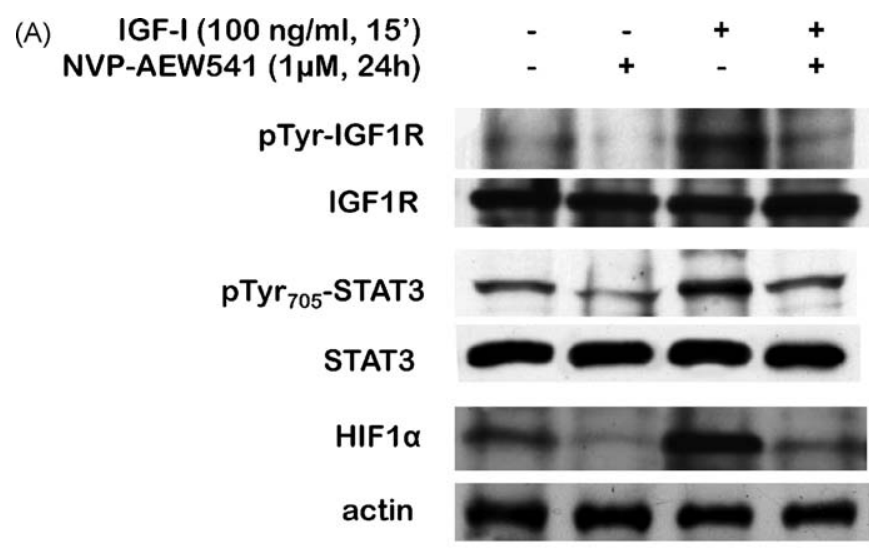

(B)

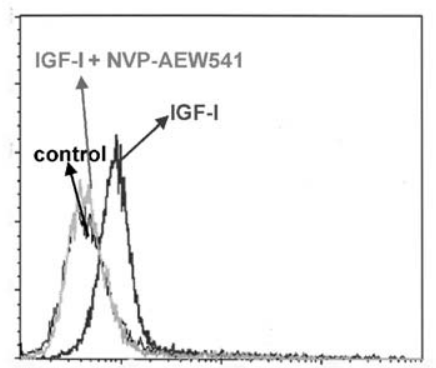

(C)

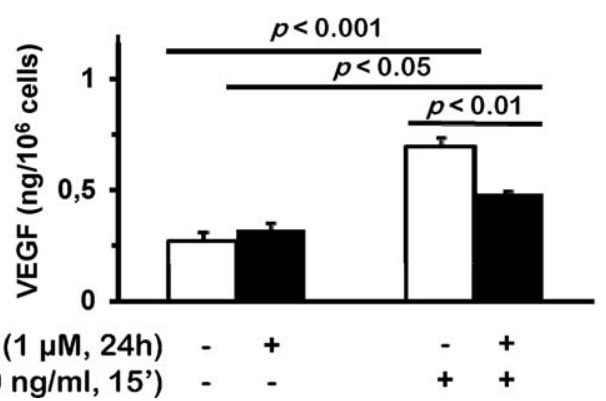

Fig. 1. Effect of IGF-I ( $100 \mathrm{ng} / \mathrm{ml}$ for $15 \mathrm{~min}$ ) and of inhibition of IGF1R tyrosine kinase activity by NVP-AEW541 ( $1 \mu \mathrm{M}$ for $24 \mathrm{~h})$ on: (A) IGF1R and STAT3 phosphorylation and IGF1R, STAT3 and HIF1 $\alpha$ protein levels; (B) HIF1 transcriptional activity; (C) VEGF release in U-87 MG human glioma cells. Mean \pm SE of 3 independent experiments. Actin levels were determined as a control for equal gel loading. 
under baseline conditions, and their intensities markedly increased following exposure to IGF-I ( $100 \mathrm{ng} / \mathrm{ml}$ for $15 \mathrm{~min})$. As expected, treatment with NVP-AEW541 ( $1 \mu \mathrm{M}$ for $24 \mathrm{~h}$, including the last 15 min with IGF-I) caused pTyr-IGF1R to revert to baseline levels. Interestingly, this treatment also reduced the levels of pTyr $_{705}$-STAT3 and of HIF1 $\alpha$, suggesting that IGF1R acts upstream of both. Total IGF1R and STAT3 protein levels were unchanged.

HIF1 transcriptional activity was also up-regulated by IGF-I, as indicated by the shift of the EGFP fluorescence peak towards higher intensity values, and inhibited by NVP-AEW541, with the fluorescence peak reverting to control values (Fig. 1B). This finding is indirectly confirmed by increased VEGF release following IGF-I treatment and by its inhibition in the presence of NVP-AEW541 (Fig. 1C).

\subsection{STAT3 inhibition blocks HIF1 activation and VEGF release}

Fig. 2A shows STAT3, pSTAT3 and HIF1 $\alpha$ levels in U-87 MG cells following transfection of the pEF-HA-STAT3F expression vector, encoding a dominant negative form of STAT3 (U-87 STAT3F), or with the "empty" vector pEF-BOS (U-87 pEF-BOS), with or without IGF-I stimulation. As expected, pSTAT3 levels were lower in U-87 STAT3F than in U-87 pEF-BOS control cells under baseline conditions; in contrast, total STAT3 levels were similar in the two cell lines, and were not modified by treatment with IGF-I. Following IGF-I exposure ( $100 \mathrm{ng} / \mathrm{ml}$ for $15 \mathrm{~min}$ ), the activated form of STAT3 was found to increase in U-87 pEF-BOS cells, while pSTAT3 levels were unaffected in U-87 STAT3F cells (Fig. 2A). Accordingly, HIF1 $\alpha$ protein levels, as well as VEGF release (Fig. 2B),

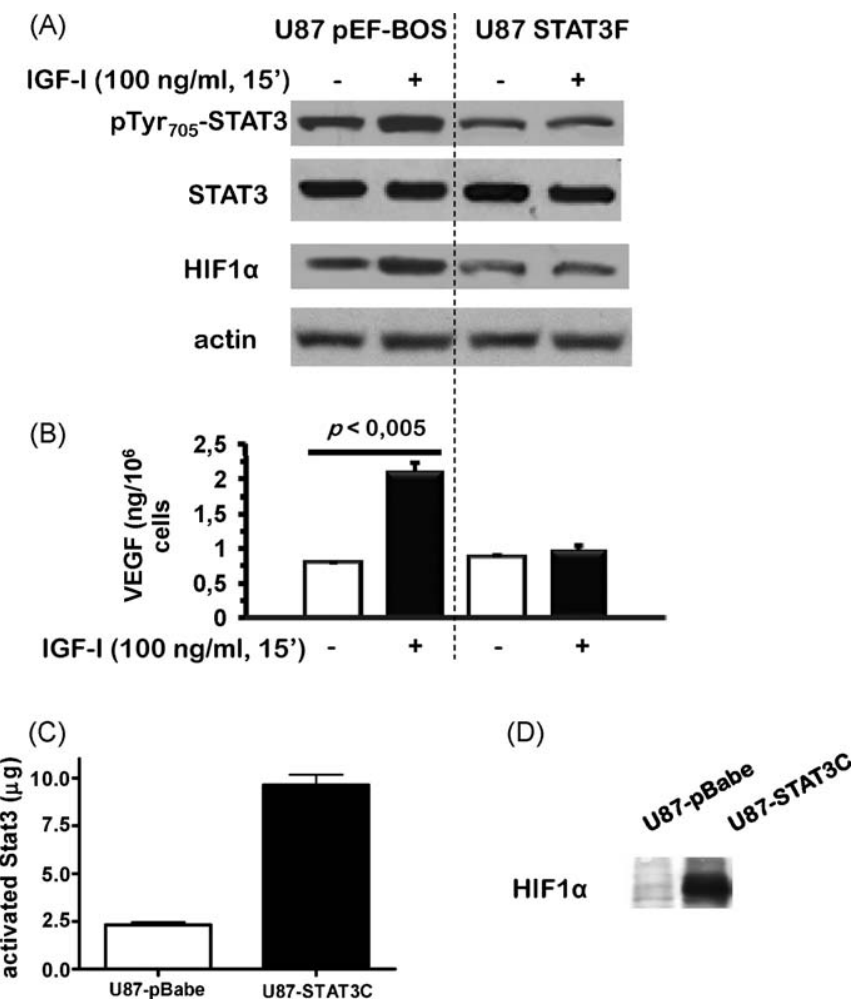

Fig. 2. Effect of IGF-I on: (A) total and phosphorylated STAT3 and HIF1 $\alpha$ protein levels and (B) VEGF release in U-87 MG cells transfected with a dominant negative form of STAT3 (U-87 STAT3F); cells transfected with the "empty" pEF-BOS vector (U-87 pEF-BOS) were used as controls. Transcriptional activity of STAT3 (panel C) and HIF1 $\alpha$ protein levels (panel D) in U-87 MG cells transfected with a constitutively active form of STAT3 (U-87 STAT3C); cells transfected with the "empty" pBabe vector (U-87 pBabe) were used as controls. Mean $\pm \mathrm{SE}$ of 3 independent experiments. Actin levels were determined as a control for equal gel loading. were also increased in U-87 pEF-BOS cells following exposure to IGF-I, whereas no increase was observed in U-87 STAT3F cells, indicating that STAT3 inhibition prevents IGF-I-induced HIF1 $\alpha$ accumulation (and HIF1 transcriptional activation). The role played by STAT3 in HIF1 $\alpha$ accumulation is confirmed by the finding that, in U87-STAT3C cells, expressing a constitutively active form of the transcription factor (Fig. 2C), HIF1 $\alpha$ protein levels are increased under normoxic conditions, as compared with U-87 pBabe cells (Fig. 2D).

\subsection{HIF1 $\alpha$ stabilization causes IGF1R activation by inducing IGF-II release}

In line with the literature [22], exposure of U-87 MG cells to $\mathrm{CoCl}_{2}(50 \mu \mathrm{M}$ for $24 \mathrm{~h}$ ) induces a marked increase in HIF1 $\alpha$ levels (Fig. $3 \mathrm{~A}$ ), due to decreased degradation by the proteasome. Under these experimental conditions, pTyr-IGF1R levels are also increased, even in the absence of added exogenous IGFs. The addition of YC-1 ( $10 \mu \mathrm{M}$ for $24 \mathrm{~h}$ ), a guanylyl cyclase activator that has been shown to decrease HIF1 $\alpha$ accumulation and HIF1 activity [27-29], reduces HIF1 $\alpha$ protein levels almost to baseline values. Interestingly, pTyr-IGF1R, but not total IGF1R, levels are also reduced by YC-1 treatment, indicating that HIF1 plays a causal role in IGF1R activation. In agreement with this finding, and with the putative role of STAT3 in the IGF1R-HIF1-IGF-II circuit, pTyr $_{705}$-STAT3 levels are increased following exposure to $\mathrm{CoCl}_{2}$ and this effect is prevented by simultaneous exposure to $\mathrm{YC}-1$. The hypothetical role of IGF-II in the circuit is confirmed by the findings reported in Fig. 3B, showing IGF-II release from U-87 MG cells following HIF1 $\alpha$ stabilization by $\mathrm{CoCl}_{2}$, an effect that is also inhibited by YC-1. These results point to IGF-II as the molecular effector actually mediating HIF1-induced IGF1R activation.

\subsection{NVP-AEW541 inhibits glioma cell growth and disrupts the autocrine loop initiated by HIF1 $\alpha$ stabilization}

Fig. 4A shows the growth curves $(0-72 \mathrm{~h})$ obtained for U-87 MG glioma cells in the absence or presence of different NVP-AEW541 concentrations, and indicates that selective inhibition of IGF1R activity decreases cell growth in a dose-dependent fashion; $\mathrm{IC}_{50}$ values extrapolated from dose response curves at 24 and $72 \mathrm{~h}$ are $2.07 \pm 0.050$ and $0.99 \pm 0.021 \mu \mathrm{M}$, respectively. In addition, NVPAEW541 ( $1 \mu \mathrm{M}$ for $24 \mathrm{~h}$ ) significantly inhibits the growth of U-87 MG cells in which HIF1 $\alpha$ has been stabilized by exposure to $\mathrm{CoCl}_{2}(50 \mu \mathrm{M}$ for $24 \mathrm{~h}$ ) (Fig. 4B). On a molecular level, under the same experimental conditions NVP-AEW541 is not only able to prevent IGF1R activation in the absence of added IGFs, but also to reduce HIF1 $\alpha$ accumulation (Fig. 5A), which suggests disruption of a positive feedback loop, very likely sustained by IGF-II release (see above). Accordingly, HIF1transcriptional activity and HIF-induced VEGF release, which are significantly increased following exposure to $\mathrm{CoCl}_{2}$, are markedly reduced by co-treatment with NVP-AEW541 (Fig. 5B and C).

\section{Discussion}

A large body of converging evidence from laboratory investigations and population studies implicates the IGF system in the development of malignancies, including gliomas [3,13]. IGF-I has been shown to affect both proliferation and migration of glioblastoma cell lines in vitro, and IGF1R inhibition has been reported to contribute to growth suppression of primary cell lines derived from human high-grade gliomas [30,31]. In contrast to other growth factor receptors, and most notably to EGFR (which is also involved in gliomagenesis), IGF1R activation in tumors is hardly ever due to gene amplification or point mutations. Rather, constitutive receptor activation is commonly caused by increased 
(A) $\mathrm{CoCl}_{2}(50 \mu \mathrm{M}, 24 \mathrm{~h})$

YC-1 $(10 \mu \mathrm{M}, 24 \mathrm{~h})$
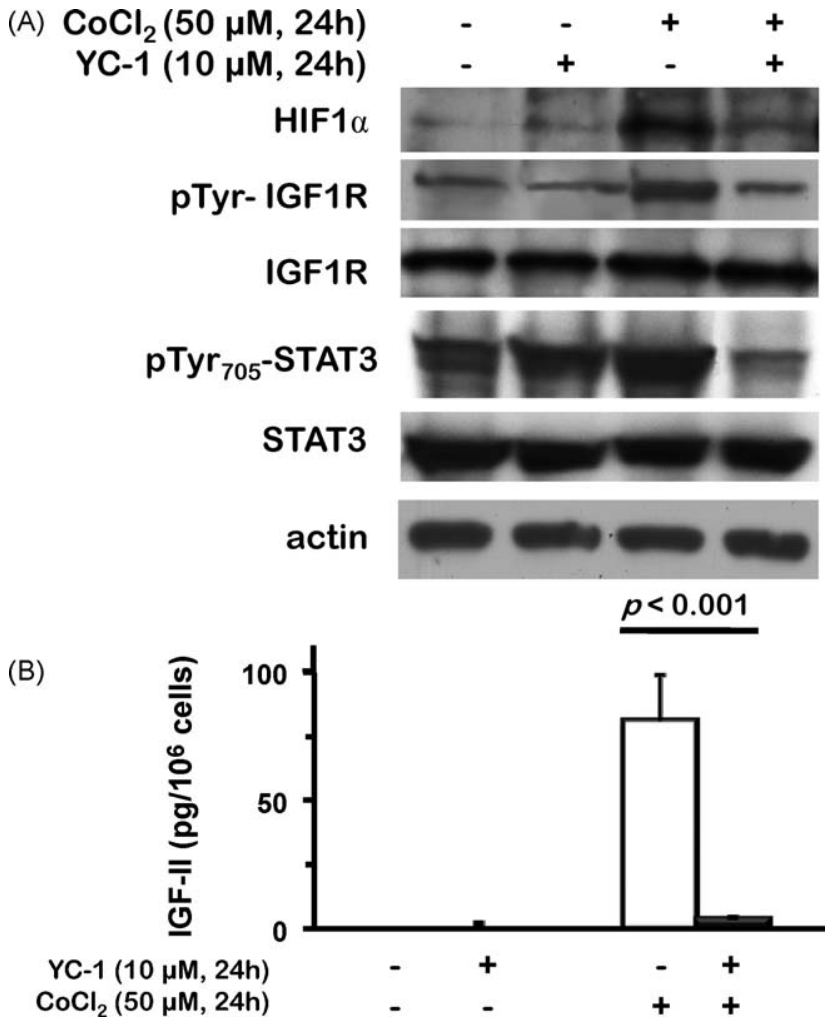

(B)

Fig. 3. Effect of exposure to the hypoxia mimic $\mathrm{CoCl}_{2}(50 \mu \mathrm{M}$ for $24 \mathrm{~h}$ ), with or without the HIF1 inhibitor YC-1 (10 $\mu \mathrm{M}$ for $24 \mathrm{~h}$ ), on: (A) HIF1 $\alpha$, IGF1R and STAT3 protein levels, and IGF1R and STAT3 phosphorylation; and (B) on the release of IGFII in U-87 MG human glioma cells. Mean \pm SE of 3 independent experiments. Actin levels were determined as a control for equal gel loading.

ligand availability, depending on a number of factors, such as decreased levels of IGF-binding proteins (IGFBPs) and/or IGF2R (a decoy receptor primarily sequestering IGF-II), or increased release of IGFs through paracrine or autocrine processes [15]. The working hypothesis underlying the present study is that autocrine activation of IGFR1 is a likely occurrence in high-grade gliomas, where hypoxic conditions frequently prevail, in spite of active neoangiogenesis, due to high proliferation rates and to the aberrant structure of newly formed vessels [32,33]. In IGF1Rexpressing cells, hypoxia may trigger a positive self-regulatory circuit mediated by HIF1 activation and subsequent release of IGFII [7], an important by-product of the circuit being increased VEGF release. If such a circuit could be demonstrated to operate in gliomas, this would constitute a powerful rationale for using one of the several recently developed IGF1R-targeting agents in the clinical management of this tumor type. To verify our hypothesis, we first demonstrated that IGF1R activation by exogenously applied IGF-I was indeed able to increase the levels of HIF1 $\alpha$, the inducible subunit controlling HIF1 transcriptional activity, in human U-87 MG glioblastoma cells under normoxic conditions; as a result, HIF1 transcriptional activity also increased, as demonstrated using a GFP-expressing reporter construct (Fig. 1B) and by a significant increase in VEGF release (Fig. 1C). All these effects were abrogated, or significantly reduced, by exposure to NVP-AEW541, a pyrrolopyrimidine derivative selectively targeting the catalytic domain of IGF1R [21]. These observations are in agreement with findings reported for other tumors/cell lines, corroborating the existence of an IGF-HIF1-VEGF axis in different tumor types $[6,11,34,35]$. Growth factor receptor activation is known to increase HIF1 $\alpha$ levels in an oxygen-independent fashion, and this effect is generally attributed to increased protein synthesis via the PI3K/Akt/mTOR pathway [36]. In the present study, the contribu-
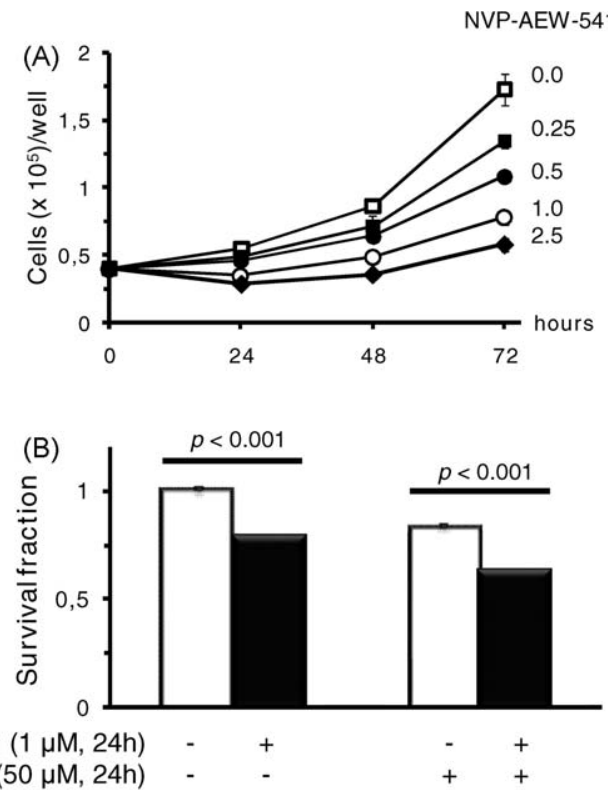

Fig. 4. Effect of NVP-AEW541 on the growth of U-87 MG human glioma cells. (A) Growth curves obtained in the presence of different NVP-AEW541 concentrations; mean \pm SE of 3 independent experiments. (B) Effect of NVP-AEW541 ( $1 \mu \mathrm{M}$ for $24 \mathrm{~h})$ on survival rates in U-87 MG cells, with or without HIF1 $\alpha$ stabilization with $\mathrm{CoCl}_{2}$ (50 $\mu \mathrm{M}$ for $24 \mathrm{~h}$ ); mean $\pm \mathrm{SE}$ of 4 independent experiments.

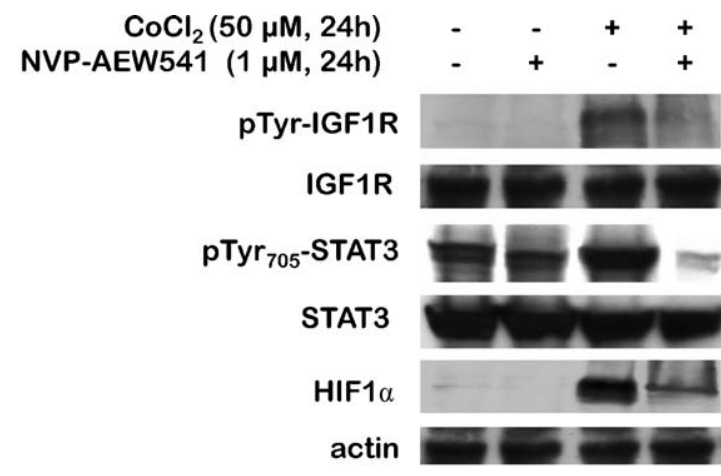

(B)
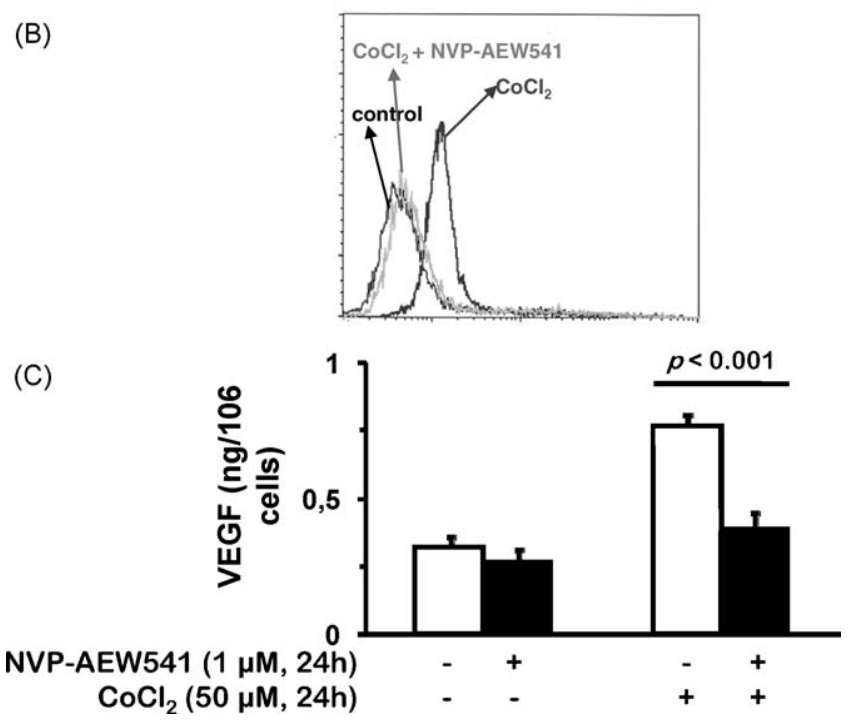

Fig. 5. Effect of NVP-AEW541: (A) HIF1 $\alpha$, IGF1R and STAT3 protein levels, and IGF1R and STAT3 phosphorylation; (B) HIF1 transcriptional activity; (C) VEGF release in U87 MG human glioma cells (A and C) or U-87 HRP/EGFP (B) cells, with or without HIF1 $\alpha$ stabilization with $\mathrm{CoCl}_{2}(50 \mu \mathrm{M}$ for $24 \mathrm{~h})$; mean $\pm \mathrm{SE}$ of 3 independent experiments. Actin levels were determined as a control for equal gel loading. 
tion of this pathway to HIF1 activation was not specifically addressed; however, it is worth noting that, in the case of IGF1R, the "canonical" model for PI3K/Akt/mTOR activation involves intracellular engagement of insulin receptor substrates (IRS) 1 and 2 to the activated IGF1R, which in turn leads to recruitment of the regulatory subunit ( $\mathrm{p} 85$ ) of $\mathrm{PI} 3 \mathrm{~K}$, followed by activation of the p110 catalytic subunit [37]. As mTOR is known to downregulate IRS-1/2 [38,39], activation of this pathway would have a selflimiting impact on HIF1 $\alpha$ synthesis. However, other signaling cascades downstream of IGF1R might lead to HIF1 activation: besides the Ras/Raf/MEK/ERK pathway, known to enhance HIF1 activity by phosphorylating the $\mathrm{C}$-terminal transactivating domain of HIF1 $\alpha$ and facilitating interactions with transcriptional coactivators [36], the JAK/STAT pathway has also been implicated as an important mediator in the IGF-dependent signaling in a number of tissues and tumor types [40]. Of note, constitutive activation of STAT3, as indicated by Tyr $_{705}$ phosphorylation, has been reported in brain tumors $[10,41]$, where it may play a major role in driving angiogenesis, but up to now its role in IGF signaling has not been extensively investigated in this tumor type. In our experimental model, constitutively active STAT3C increases HIF1 $\alpha$ protein levels, thereby supporting that STAT3-dependent signaling pathways contribute to HIF1 activation. In addition, our data indicate that in U-87 MG glioblastoma cells STAT3 acts downstream of IGF1R activation and upstream of HIF1, based on the ability of DNSTAT3F to prevent the increase in HIF1 $\alpha$ normally observed upon exposure to exogenous IGF-I (Fig. 2). Mechanistically, the effect of STAT3 on HIF1 $\alpha$ levels seems to depend on a variation of the canonical IGF1R-PI3K-AKT-mTOR model outlined above: STAT3 can be recruited by the IGF1R independent of IRS1/2 [12] and subsequently regulates Akt1 expression [42], which may increase HIF1 $\alpha$ protein synthesis. In addition, STAT3 has been shown to increase HIF1 $\alpha$ mRNA levels [43] and to stabilize HIF1 $\alpha$ by inhibiting its binding to the Von Hippel-Lindau protein [44]. Finally STAT3 and HIF1 $\alpha$ have been shown to form a multiprotein complex with transcription coactivators $\mathrm{CBP} / \mathrm{p} 300$ and Ref-1/Ape, that binds to the VEGF promoter and regulates gene expression [45].

Having established that an IGF-(STAT3)-HIF1 axis operates in U87 MG cells, we then proceeded to investigate the existence of an autocrine circuit involving these same players. To this aim, we induced HIF1 activation independent of IGF1R stimulation, by exposing the cells to $\mathrm{CoCl}_{2}$ to stabilize HIF1 $\alpha$, and observed that under these experimental conditions IGF1R was activated in the absence of exogenously added ligands. Importantly, such activation was prevented by YC-1, a guanylyl cyclase activator that is also known to inhibit HIF1 $\alpha$ accumulation and HIF1 transcriptional activity [27-29], indicating that HIF1 activation is an obligatory step in the circuit (Fig. 3). Incidentally, this experiment also confirmed the involvement of STAT3 in this signaling axis, based on the increase in $\mathrm{pTyr}_{705}$-STAT3 induced by $\mathrm{COCl}_{2}$ and the ability of YC-1 to prevent it. The observed cell-autonomous activation of IGF1R strongly suggests that autocrine stimulation of the receptor occurs following HIF1-induced release of specific ligand(s). Indeed, $\mathrm{CoCl}_{2}$-stimulated cells were found to release IGF-II, the product of a recognized HIF1 target gene; IGF-II release was significantly suppressed by YC-1 at the same concentration that was shown to block IGF1R and STAT3 activation. Similar autocrine regulatory loops have been described in cell lines and xenografts derived from other tumor types, where they appear to play a key role in tumor cell survival and angiogenesis $[1,2,11]$. It is important to underline that the circuit can be set in motion by different stimuli besides IGF1R stimulation, including HIF1 activation by hypoxia and/or growth factors and STAT3 activation by growth factor or cytokine receptors. Thus, in principle, strategies directed at any one step of the circuit, including targeting HIF1 activity with small molecules or nucleotide-based agents $[17,18]$, or STAT3 activation using inhibitors of upstream kinases [46,47] should have a disruptive effect on the whole circuit. As antibodies and small molecule tyrosine kinase inhibitors targeting IGFR1 are by far the most advanced in the clinic $[48,49]$, in the present study we decided to use NVP-AEW541 to verify how blocking this step in the circuit would affect the survival and pro-angiogenic features of glioblastoma cells. Recent data by Moser et al. indicate that in IGF-Istimulated pancreatic cancer cell inhibition of IGF1R activation by NVP-AEW541 was accompanied by decreased STAT3 phosphorylation and led to reduced IGF-dependent growth [11]; inhibition of tumor growth and angiogenesis was also observed in mice bearing pancreatic cancer xenografts following NVP-AEW541 treatment in vivo. In U-87 MG cells, we found that NVP-AEW541 inhibited cell growth in a time and concentration-dependent fashion (Fig. 4A). Cell survival was also inhibited in U-87 MG cells following HIF1 $\alpha$ stabilization with $\mathrm{CoCl}_{2}$ (Fig. 4B); however, the extent of growth inhibition by NVP-AEW541 was not significantly affected by the HIF1 $\alpha$-stabilizing treatment, even though NVP-AEW541 was indeed able to reduce $\mathrm{HIF} 1 \alpha$ levels in $\mathrm{CoCl}_{2}$-treated cells (Fig. 5A). So, from our results it would appear that, while IGF1R receptor inhibition has a distinct effect on U-87 MG cell survival, the fact that HIF1 $\alpha$ stabilization is also significantly prevented does not result in any added benefit in terms of growth inhibition, which therefore seems to depend on disruption of IGF1R signaling as a whole rather than solely on disruption of the described IGF(STAT3)-HIF1 circuit. This is not altogether surprising, given the multiple signaling pathways downstream of IGF1R that might contribute to cell survival and that are curtailed by NVP-AEW541, whereas blocking HIF1 $\alpha$ accumulation (by RNA interference) has been shown to cause only marginal cytotoxicity in glioma cells in vitro [50]. In contrast, blocking IGFR1 activation caused by HIF1 stabilization resulted in a significant decrease in VEGF release in NVP-AEW541-treated cells, whereas no effect was observed on VEGF release under baseline conditions (i.e. when HIF1 $\alpha$ levels were not artificially elevated by $\mathrm{CoCl}_{2}$ ). Therefore, based on these results, activation of the circuit does seem to be crucial in maintaining the angiogenic potential of U-87 MG cells, as assessed by their ability to release significant amounts of VEGF. To support this conclusion, silencing HIF1 $\alpha$ by RNA interference was shown to inhibit the in vivo growth of xenografts obtained from the same glioma cell lines, the survival of which was only weakly impaired in vitro [50], which strongly suggests that the pro-angiogenic effect of HIF1 is crucial for the in vivo growth of glial tumors, rather than its ability to directly stimulate intracellular pro-survival mechanisms. On the other hand, the fact that blocking IGFR1 is able to curb HIF1 activation should not be overlooked, in view of its possible implications for the design of drug combinations. HIF1 is well known for its role as a resistance factor impairing the activity of a number of cytotoxic agents in several tumor types [51,52]. More specifically, in vitro studies on glioma cell lines indicate that HIF1 activation significantly impairs the cytotoxicity of two of the very few agents that show some effect against this tumor type, namely temozolomide and BCNU $[53,54]$, and this scenario is very likely to occur in vivo in glioblastomas, as mentioned above. Thus, blocking IGF1R signaling with NVP-AEW541, or with one of the several agents targeting this receptor that are currently undergoing clinical trials, could be successfully exploited in the clinical management of gliomas for its double effect on tumor angiogenesis and on drug sensitivity of hypoxic cells.

To conclude, our data support the hypothesis that in U-87 MG human glioma cells a self-amplifying autocrine loop exists, that can be activated by IGFs and hypoxia, as well as by signaling pathways that impinge on STAT3, and that can lead to enhanced angiogenic potential via an increase in VEGF release (Fig. 6). Inhibition of IGF1R tyrosine kinase activity, e.g. by using NVPAEW541, can disrupt such a circuit, even when the primary trigger 


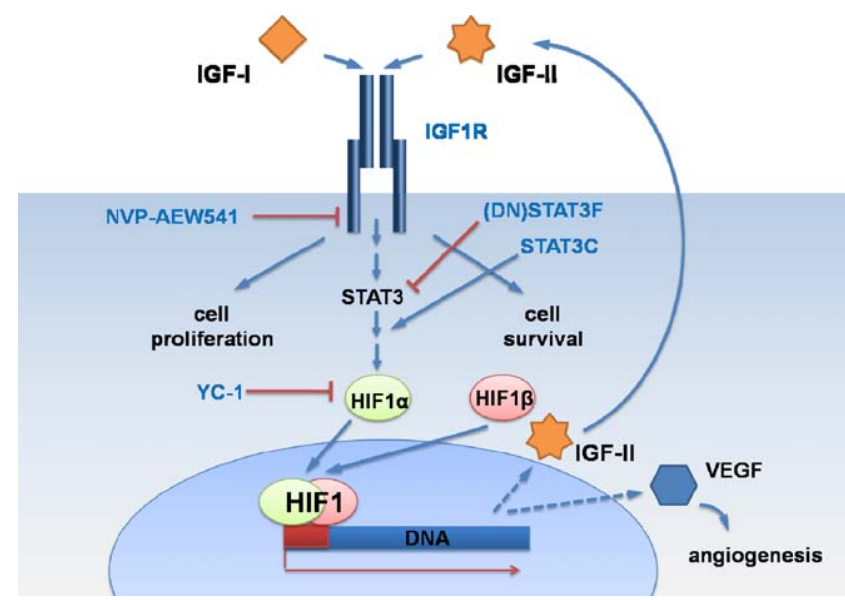

Fig. 6. Proposed model for a self-regulatory circuit involving the IGF system, STAT3 and HIF1, and regulating cell proliferation and angiogenesis in human U-87 MC glioma cells.

for circuit activation is not the engagement of the receptor by its ligands, thereby representing a potential therapeutic approach, especially for the management of solid tumors characterized by hypoxic regions.

\section{References}

[1] Thomas R, Kim M. A HIF-1alpha-dependent autocrine feedback loop promotes survival of serum-deprived prostate cancer cells. Prostate 2009;69:263-75.

[2] Stoeltzing O, Liu W, Reinmuth N, Fan F, Parikh A, Bucana C, et al. Regulation of hypoxia-inducible factor-1alpha, vascular endothelial growth factor, and angiogenesis by an insulin-like growth factor-I receptor autocrine loop in human pancreatic cancer. Am J Pathol 2003;163:1001-11.

[3] Pollak M. Insulin and insulin-like growth factor signalling in neoplasia. Nat Rev Cancer 2008;8:915-28.

[4] Werner H, Bruchim I. The insulin-like growth factor-I receptor as an oncogene. Arch Physiol Biochem 2009;115:58-71.

[5] Yee Koh M, Spivak-Kroizman T, Powis G. HIF-1 regulation: not so easy come, easy go. Trends Biochem Sci 2008;33:526-34.

[6] Fukuda R, Hirota K, Fan F, Jung Y, Ellis L, Semenza G. Insulin-like growth factor 1 induces hypoxia-inducible factor 1-mediated vascular endothelial growth factor expression, which is dependent on MAP kinase and phosphatidylinosito 3-kinase signaling in colon cancer cells. J Biol Chem 2002;277:38205-11.

[7] Feldser D, Agani F, Iyer N, Pak B, Ferreira G, Semenza G. Reciprocal positive regulation of hypoxia-inducible factor 1 alpha and insulin-like growth factor 2 . Cancer Res 1999;59:3915-8.

[8] Yu H, Jove R. The STATs of cancer-new molecular targets come of age. Nat Rev Cancer 2004;4:97-105.

[9] Garcia R, Bowman T, Niu G, Yu H, Minton S, Muro-Cacho C, et al. Constitutive activation of Stat3 by the Src and JAK tyrosine kinases participates in growth regulation of human breast carcinoma cells. Oncogene 2001;20:2499-513.

[10] Brantley E, Nabors L, Gillespie G, Choi Y, Palmer C, Harrison K, et al. Loss of protein inhibitors of activated STAT-3 expression in glioblastoma multiforme tumors: implications for STAT-3 activation and gene expression. Clin Cancer Res 2008; 14:4694-704.

[11] Moser C, Schachtschneider P, Lang S, Gaumann A, Mori A, Zimmermann J, et al Inhibition of insulin-like growth factor-I receptor (IGF-IR) using NVP-AEW541, a small molecule kinase inhibitor, reduces orthotopic pancreatic cancer growth and angiogenesis. Eur J Cancer 2008;44:1577-86.

[12] Zhang W, Zong C, Hermanto U, Lopez-Bergami P, Ronai Z, Wang L. RACK1 recruits STAT3 specifically to insulin and insulin-like growth factor 1 receptors for activation, which is important for regulating anchorage-independent growth. Mol Cell Biol 2006;26:413-24.

[13] Trojan J, Cloix J, Ardourel M, Chatel M, Anthony D. Insulin-like growth factor type I biology and targeting in malignant gliomas. Neuroscience 2007;145: 795-811.

[14] Pollak M. Targeting insulin and insulin-like growth factor signalling in oncology. Curr Opin Pharmacol 2008;8:384-92.

[15] Sachdev D, Yee D. Disrupting insulin-like growth factor signaling as a potential cancer therapy. Mol Cancer Ther 2007;6:1-12.

[16] Semenza G. Targeting HIF-1 for cancer therapy. Nat Rev Cancer 2003;3:72132.

[17] Semenza G. Evaluation of HIF-1 inhibitors as anticancer agents. Drug Discov Today 2007;12:853-9.

[18] Greenberger L, Horak I, Filpula D, Sapra P, Westergaard M, Frydenlund H, et al. A RNA antagonist of hypoxia-inducible factor-1alpha, EZN-2968, inhibits tumor cell growth. Mol Cancer Ther 2008;7:3598-608.
[19] Koh M, Spivak-Kroizman T, Venturini S, Welsh S, Williams R, Kirkpatrick D, et al. Molecular mechanisms for the activity of PX-478, an antitumor inhibitor of the hypoxia-inducible factor-1alpha. Mol Cancer Ther 2008;7:90-100.

[20] Zumkeller W, Westphal M. The IGF/IGFBP system in CNS malignancy. Mol Pathol 2001;54:227-9.

[21] García-Echeverría C, Pearson M, Marti A, Meyer T, Mestan J, Zimmermann J, et al. In vivo antitumor activity of NVP-AEW541-A novel, potent, and selective inhibitor of the IGF-IR kinase. Cancer Cell 2004;5:231-9.

[22] Yuan Y, Hilliard G, Ferguson T, Millhorn D. Cobalt inhibits the interaction between hypoxia-inducible factor-alpha and von Hippel-Lindau protein by direct binding to hypoxia-inducible factor-alpha. J Biol Chem 2003;278: 15911-6.

[23] Mizushima S, Nagata S. pEF-BOS, a powerful mammalian expression vector. Nucleic Acids Res 1990;18:5322.

[24] Nakajima K, Yamanaka Y, Nakae K, Kojima H, Ichiba M, Kiuchi N, et al. A central role for Stat3 in IL-6-induced regulation of growth and differentiation in M1 leukemia cells. EMBO J 1996;15:3651-8.

[25] Bromberg J, Wrzeszczynska M, Devgan G, Zhao Y, Pestell R, Albanese C, et al. Stat3 as an oncogene. Cell 1999;98:295-303.

[26] Cao Y, Li C, Moeller B, Yu D, Zhao Y, Dreher M, et al. Observation of incipient tumor angiogenesis that is independent of hypoxia and hypoxia inducible factor-1 activation. Cancer Res 2005;65:5498-505.

[27] Kim H, Yeo E, Chun Y, Park J. A domain responsible for HIF-1alpha degradation by YC-1, a novel anticancer agent. Int J Oncol 2006;29:255-60.

[28] Li S, Shin D, Chun Y, Lee M, Kim M, Park J. A novel mode of action of YC-1 in HIF inhibition: stimulation of FIH-dependent p300 dissociation from HIF-1 \{alpha\}. Mol Cancer Ther 2008;7:3729-38.

[29] Sun H, Liu Y, Huang Y, Pan S, Huang D, Guh J, et al. YC-1 inhibits HIF-1 expression in prostate cancer cells: contribution of Akt/NF-kappaB signaling to HIF-1alpha accumulation during hypoxia. Oncogene 2007;26:3941-51.

[30] Schlenska-Lange A, Knüpfer H, Lange T, Kiess W, Knüpfer M. Cell proliferation and migration in glioblastoma multiforme cell lines are influenced by insulinlike growth factor I in vitro. Anticancer Res 2008;28:1055-60.

[31] Carapancea M, Cosaceanu D, Budiu R, Kwiecinska A, Tataranu L, Ciubotaru V, et al. Dual targeting of IGF-1R and PDGFR inhibits proliferation in high-grade gliomas cells and induces radiosensitivity in JNK-1 expressing cells. J Neurooncol 2007;85:245-54

[32] Brat D, Mapstone T. Malignant glioma physiology: cellular response to hypoxia and its role in tumor progression. Ann Intern Med 2003;138:659-68.

[33] Kaur B, Khwaja F, Severson E, Matheny S, Brat D, Van Meir E. Hypoxia and the hypoxia-inducible-factor pathway in glioma growth and angiogenesis. Neuro Oncol 2005;7:134-53.

[34] Beppu K, Nakamura K, Linehan W, Rapisarda A, Thiele C. Topotecan blocks hypoxia-inducible factor-1alpha and vascular endothelial growth factor expression induced by insulin-like growth factor-I in neuroblastoma cells. Cancer Res 2005;65:4775-81.

[35] Slomiany M, Rosenzweig S. Hypoxia-inducible factor-1-dependent and -independent regulation of insulin-like growth factor-1-stimulated vascular endothelial growth factor secretion. J Pharmacol Exp Ther 2006;318:666-75.

[36] Semenza G. Signal transduction to hypoxia-inducible factor 1. Biochem Pharmacol 2002;64:993-8

[37] Chitnis M, Yuen J, Protheroe A, Pollak M, Macaulay V. The type 1 insulin-like growth factor receptor pathway. Clin Cancer Res 2008;14:6364-70.

[38] Harrington L, Findlay G, Lamb R. Restraining PI3K: mTOR signalling goes back to the membrane. Trends Biochem Sci 2005;30:35-42.

[39] Huang J, Manning B. A complex interplay between Akt, TSC2 and the two mTOR complexes. Biochem Soc Trans 2009;37:217-22.

[40] Himpe E, Kooijman R. Insulin-like growth factor-I receptor signal transduction and the Janus Kinase/Signal Transducer and Activator of Transcription (JAKSTAT) pathway. Biofactors 2009; 35:76-81.

[41] Schaefer L, Ren Z, Fuller G, Schaefer T. Constitutive activation of Stat3alpha in brain tumors: localization to tumor endothelial cells and activation by the endothelial tyrosine kinase receptor (VEGFR-2). Oncogene 2002;21:205865.

[42] Xu Q, Briggs J, Park S, Niu G, Kortylewski M, Zhang S, et al. Targeting Stat3 blocks both HIF-1 and VEGF expression induced by multiple oncogenic growth signaling pathways. Oncogene 2005;24:5552-60.

[43] Jung J, Lee H, Cho I, Chung D, Yoon S, Yang Y, et al. STAT3 is a potential modulator of HIF-1-mediated VEGF expression in human renal carcinoma cells. FASEB J 2005;19:1296-8.

[44] Jung J, Kim H, Lee C, Shin Y, Kim Y, Kang G, et al. STAT3 inhibits the degradation of HIF-1alpha by pVHL-mediated ubiquitination. Exp Mol Med 2008;40:47985.

[45] Gray M, Zhang J, Ellis L, Semenza G, Evans D, Watowich S, et al. HIF-1alpha, STAT3, CBP/p300 and Ref-1/APE are components of a transcriptional complex that regulates Src-dependent hypoxia-induced expression of VEGF in pancreatic and prostate carcinomas. Oncogene 2005;24:3110-20.

[46] Burger R, Le Gouill S, Tai Y, Shringarpure R, Tassone P, Neri P, et al. Janus kinase inhibitor INCB20 has antiproliferative and apoptotic effects on human myeloma cells in vitro and in vivo. Mol Cancer Ther 2009;8:26-35.

[47] Caceres-Cortes J. A potent anti-carcinoma and anti-acute myeloblastic leukemia agent, AG490. Anticancer Agents Med Chem 2008;8:717-22.

[48] Rodon J, DeSantos V, Ferry RJ, Kurzrock R. Early drug development of inhibitors of the insulin-like growth factor-I receptor pathway: lessons from the first clinical trials. Mol Cancer Ther 2008;7:2575-88. 
[49] Weroha S, Haluska P. IGF-1 receptor inhibitors in clinical trials-early lessons. J Mammary Gland Biol Neoplasia 2008;13:471-83.

[50] Gillespie D, Whang K, Ragel B, Flynn J, Kelly D, Jensen R. Silencing of hypoxia inducible factor-1alpha by RNA interference attenuates human glioma cell growth in vivo. Clin Cancer Res 2007:13:2441-8.

[51] Sullivan R, Paré G, Frederiksen L, Semenza G, Graham C. Hypoxia-induced resistance to anticancer drugs is associated with decreased senescence and requires hypoxia-inducible factor-1 activity. Mol Cancer Ther 2008;7:196173.
[52] Unruh A, Ressel A, Mohamed H, Johnson R, Nadrowitz R, Richter E, et al. The hypoxia-inducible factor- 1 alpha is a negative factor for tumor therapy. Oncogene 2003;22:3213-20.

[53] Yang D, Chen S, Yang Y, Ju T, Xu J, Hsu C. Carbamoylating chemoresistance induced by cobalt pretreatment in C6 glioma cells: putative roles of hypoxiainducible factor-1. Br J Pharmacol 2004;141:988-96.

[54] Monti E, Terni F, Mettifogo F, Gariboldi MB. IGF-IR inhibition modulates HIF$1 \alpha$ expression/activity and enhances the cytotoxicity of temozolomide in human glioblastoma cells. Clin Cancer Res 2005;11:9134. 